\title{
Recent Advances of Multi-Stimuli-Responsive Drug Delivery Systems for Cancer Therapy
}

\author{
Jia Wen and Shiguo Sun* \\ Shaanxi Key Laboratory of Natural Products \& Chemical Biology, Northwest Agriculture and Forestry University, China
}

Submission: March 01, 2017; Published: April 20, 2017

"Corresponding author: Shiguo Sun, Shaanxi Key Laboratory of Natural Products \& Chemical Biology, College of Chemistry \& Pharmacy Northwest Agriculture and Forestry University, China, Email: sunsg@nwsuaf.edu.cn

Abstract

Chemotherapy remains as a major treatment modality for cancer nowadays however, it is often associated with toxicity issues. To solve this problem, constructing stimuli-responsive drug delivery system (DDS) which can deliver therapeutic agents and control the release of them in a smart manner is an effective way. Among them, multi-stimuli-responsive DDS has attracted enormous interest owing to their more precise release of drugs in complicated blood circulation and pathological environment. Thus, the main focus of this review is to highlight the progresses on the design of multi-stimuli-responsive DDS for cancer therapy in the past year.

Keywords: Drug delivery system; Stimuli-responsive; Cancer therapy

\section{Introduction}

Nowadays, cancer with an increasing morbidity at an alarming rate is still a major cause of death worldwide [1]. And the most common cancer treatment method is conventional chemotherapy which is based on highly potent anticancer drugs. However, the harmful side-effects due to the nonspecific uptake by normal tissues/organs limit their further applications [2]. To conquer these limitations, an effective way is to construction stimuli-responsive drug delivery system (DDS) which can deliver therapeutic agents and control the release of them in a smart manner [3]

One of the biggest advantages of an ideal stimuli-responsive DDS is the precise control of drug release in response to exogenous or endogenous stimuli. However, most of single or dual-stimuli-responsive DDSs are easily affected by complex external factors and suffered the problem of low release accuracy and some side effects [4]. To solve this problem, multi-stimuliresponsive DDSs have been developed for more precise release in complicated blood circulation and pathological environment [5]. In this mini review, we will summarize the progress and research efforts made by different research groups on designing and constructing multi-stimuli-responsive DDSs for cancer therapy in the past year.

\section{Discussion}

In the past year, polymers with unique sensitivity are mostly used for the construction of multi-stimuli-responsive
DDSs. For example, Wang's group [6] designed a triple-stimuliresponsive DDS based on graft copolymer assembly. The graft copolymer was consisted of thermo-responsive tetraethylene glycolyl poly (trimethylene carbonate) as backbone and lightsensitive poly(2-nitrobenzyl methacrylate) as side chain linked by an intervening disulfide bond. In aqueous solution, the polymer can self-assemble into micelle loading drugs. And the drug could be release respond to temperature, reducing agent and light. Analogously, Cao et al. [7] demonstrated a multi-stimuli-responsive nanogels for selective release of simultaneously encapsulated hydrophobic and hydrophilic cargos in a spatiotemporally controlled manner. The nanogel was composed of hydrophilic $\mathrm{pH}$ - and thermo responsive poly(2-(dimethylamino)ethyl methacrylate) and hydrophobic photocleavable o-nitrobenzyl linkage. The hydrophobic cargos were noncovalently encapsulated into lipophilic interiors of the nanogels, while the hydrophilic cargos were chemically linked to the nanogel precursor polymer poly(2-(dimethylamino) ethyl methacrylate) through a redox-cleavable disulfide junction. For these dual-loaded nanogels, hydrophobic cargos can be released in response to temperature, $\mathrm{pH}$, and UV light, while the hydrophilic cargos can be released in response to redox reagent. Hu et al. [8] synthesized a core-crosslinked poly(thiolether ester) (PTE) micelles with ROS-, GSH- and pHsensitivity for intracellular drug delivery. PTE with abundant hydroxyl groups in the side chains were facilely synthesized 
by the thiol-ene/thiol-expoxy polymerization of ethanedithiol (EDT) and glycidylmethacrylate (GMA). Then, poly (ethylene glycol) (PEG) and lipoic acid (LA) were grafted onto the PTE backbone to obtain PTE-g-PEG-LA graft copolymers, which were subsequently converted to core-crosslinked nanoparticles in aqueous media. The obtained core-crosslinked PTE-g-PEGLA micelles could serve as drug delivery vehicle for effective loading of anticancer drug and triggered release of the loaded drug in response to various micro-environmental conditions (ROS-abundant, acidic or reductive environment) thosetypically existed in cancer cells. Similarly using polymer, Zhao's et al. [9] reported a multi-stimuli-responsive nanoparticles based on the co-assembly of a 3-arm star quaterpolymer with a near-infrared (NIR) photo thermal agent and chemotherapeutic compound. The nanoparticles can exhibit NIR light/pH/reduction-responsive drug release and intracellular drug translocation in cancer cells. Later, Li et al. [10] fabricated a $\mathrm{pH}$, temperature and reduction multi-stimuli responsive polymeric microsphere via distillationprecipitation polymerization technique, with or without the hollow layer between their $\mathrm{pH}$ and reduction responsive poly(methacrylic acid) (PMAA) cores and temperature sensitive poly(N-isopropylacrylamide) (PNIPAM) shells. Owing to the middle hollow layer between their $\mathrm{pH}$ and reduction responsive PMAA cores and temperature sensitive PNIPAM shells, the PMAA/PNIPAM yolk/shell microspheres possessed the higher drug loading capacity and better tumor microenvironmentresponsive controlled release performance. Most recently, Sui's et al. [11] synthesized a multiple stimuli-responsive SN38 prodrug, named PEG-S-S-SN38, by conjugating PEG (MW: 2000) and SN38 with disulfide bonds and carbonic ester linkages as linkers for efficient delivery of SN38. The amphiphilic PEG-SS-SN38, with a high SN38 loading content, could self-assemble into nanoparticles and the release of SN38 was very slowly at physiological $\mathrm{pH}$, while quickly in the presence of glutathione (GSH), esterase and $\mathrm{H}_{2} \mathrm{O}_{2}$, all of which are abundant in the cytoplasm of cancer cells.

Apart from polymers, inorganic nanomaterial is also a good candidate. For example, Zhang's et al. [12] utilized a kind of NIR resonant material-hollow mesoporous copper sulfide nanoparticles (HMCuS NPs) to encapsulate doxorubicin (DOX). And the outer surface of HMCuS NPs was capped with multifunctional hyaluronic acid (HA) simultaneously as smart gatekeeper as well as tumor targeting moiety. In the tumor acidic lysosome, the enzymatic degradation of HA took off the protective capping, then NIR irradiation and acidic $\mathrm{pH}$ further enhanced the DOX release. Later, Timin et al. [13] designed a hybrid inorganic/ organic capsules enabling multimodal triggering by physical (UV light, ultrasound) and chemical (enzymatic treatment) stimuli. The UV- and ultrasound response was achieved by a synergetic combination of $\mathrm{TiO}_{2}$ and $\mathrm{SiO}_{2}$ nanostructures which were in situ deposited into the polymer shell of microcapsules during sol-gel synthesis. At the same time, these hybrid capsules were consisted of degradable polypeptides and polysaccharides and thus could be decomposed in response to enzymatic reaction. Most recently, our group [14] designed and prepared a triple-stimuli (reduced $\mathrm{GSH}, \mathrm{pH}$ and light irradiation) responsive system based on cerium oxide nanoparticles $\left(\mathrm{CeO}_{2} \mathrm{NPs}\right)$ coated mesoporous silica nanoparticles (MSNs). Upon entering into cancer cells, both high concentration of intracellular GSH and low $\mathrm{pH}$ environment would reduce $\mathrm{CeO}_{2}$ NPs to cerium ions, accompanied with the degradation of $\mathrm{CeO}_{2} \mathrm{NPs}$ and the conformational change of HP under light irradiation, the pore entrances were re-exposed and preloaded DOX are thus released from the nanocarrier, resulting in a contrast fluorescence enhancement. Meanwhile, $10_{2}$ generated from HP for potential photodynamic therapy (PDT) upon light irradiation. In comparison, not much influence can be observed for normal cells.

\section{Conclusion}

Although multi-stimuli-responsive DDSs are remarkably superior over other DDSs for drug delivery, there also exist drawbacks currently limiting their practical application in the clinical setting. To realize their real applications in human bodies in future, several points need to be taken into consideration. First of all, enhancing the biodegradability of DDSs. Biodegradation should not only modulate the release of drugs for a desired period of time, but also enable the removal of the empty DDS after drug release. Secondly, making the size of DDSs less than $200 \mathrm{~nm}$ in diameter. The ability to configure dimensions can facilitate cellular uptake of DDSs through a receptor-mediated endocytosis to cross cell membranes, thus significantly increasing their circulation time in blood. Thirdly, prolonging circulation in the bloodstream by surface modification of the DDSs, which will enhance the deliverable capability of DDSs to the diseased site, thus reducing the side effects.

\section{Acknowledgement}

This work was financially supported by the Scientific Research Foundation of Northwest A \& F University (Z111021103 and Z111021107), the National Natural Science Foundation of China (No. 21472016, 21272030 and 21476185).

\section{Conflict of Interest}

The authors declare no competing economic interests.

\section{References}

1. Chen Yj, Zhang H, Xiaoqing C, JianboJi, Shuwang H, (2016) Multifunctional mesoporous silica nanocarriers for stimuli-responsive target delivery of anticancer drugs. Rsc Adv 6(94): 92073-92091.

2. Yang K, Chang YC, Wen J, Lu YC, Pei YX, et al. (2016) Supramolecular vesicles based on complex of trp-modified pillar [5] arene and galactose derivative for synergistic and targeted drug delivery. Chem. Mater 28(7): 1990-1993.

3. Pedron S, van Lierop S, Horstman P, Penterman R, D J Broer, et al. (2011) Stimuli responsive delivery vehicles for cardiac microtissue transplantation. Adv. Funct. Mater 21(9): 1624-1630.

4. Karimi M, Ghasemi A, Sahandi Zangabad P, Rahighi R, Moosavi Basri SM, et al. (2016) Smart micro/nanoparticles in stimulus-responsive 
drug/gene delivery systems. Chem Soc Rev 45(5): 1457-1501.

5. Jiaming Zhuang,a Mallory R. Gordon,a Judy Ventura,a Longyu Lia and S. Thayumanavan (2013) Multi-stimuli responsive macromolecules and their assemblies. Chem Soc Rev 42(17): 7421-7435.

6. Mengmeng Xie, Lin Yu, Zhao Li, Zhen Zheng, Xinling Wang (2016) Synthesis and character of novel polycarbonate for constructing biodegradable multi-stimuli responsive delivery system. J Polym Sci Pol Chem 54(22): 3583-3592.

7. Cao ZQ Zhou XT, Wang GJ (2016) Selective release of hydrophobic and hydrophilic cargos from multi-stimuli-responsive nanogels. Acs Appl Mater Inter 8(42): 28888-28896.

8. Hu Y, Deng M, Yang H, Chen L, Xiao C, et al. (2017) Multi-responsive core-crosslinked poly (thiolether ester) micelles for smart drug delivery. Polymer 110: 235-241.

9. Nan X, Zhu AJ, Luo HH, Ke HT, Chen HB, Zhao YL (2016) Rational design of multi-stimuli-responsive nanoparticles for precise cancer therapy. Acs Nano 10(6): 5947-5958.

This work is licensed under Creative Commons Attribution 4.0 License DOI: $10.19080 /$ CTBEB.2017.03.555607
10. Li JG, Zeng J, Jia X, Liu L, TZhou, et al. (2016) pH, temperature and reduction multi-responsive polymeric microspheres as drug delivery system for anti-tumor drug: Effect of middle hollow layer between $\mathrm{pH}$ and reduction dual-responsive cores and temperature sensitive shells. J. Taiwan Inst. Chem. DOI: org/10.1016/j.jtice.2016.07.013.

11. Liu X, Huang Q, Yang CX, Zhang QZ, Chen W, et al. (2017) A multi-stimuli responsive nanoparticulate SN38 prodrug for cancer chemotherapy. J Mater Chem B 5(4): 661-670.

12. Feng QH, Zhang YY, Zhang WX, Shan XN, Yuan Y J, et al. (2016) Tumor-targeted and multi-stimuli responsive drug delivery system for near-infrared light induced chemo-phototherapy and photoacoustic tomography. Acta Biomaterialia 38: 129-142.

13. Timin AS, Muslimov AR, Lepik KV, Saprykina NN, Sergeev VS, et al. (2016) Triple-responsive inorganic-organic hybrid microcapsules as a biocompatible smart platform for the delivery of small molecules. J Mater Chem B 4(45): 7270-7282.

14. Wen J, Yang K, Xu YQ, Li HJ, Liu FY, et al. (2016) Construction of a triple-stimuli-responsive system based on cerium oxide coated mesoporous silica nanoparticles. Sci Rep-Uk 6: 38931.

\section{Your next submission with Juniper Publishers will reach you the below assets}

- Quality Editorial service

- Swift Peer Review

- Reprints availability

- E-prints Service

- Manuscript Podcast for convenient understanding

- Global attainment for your research

- Manuscript accessibility in different formats

( Pdf, E-pub, Full Text, Audio)

- Unceasing customer service

Track the below URL for one-step submission https://juniperpublishers.com/online-submission.php 